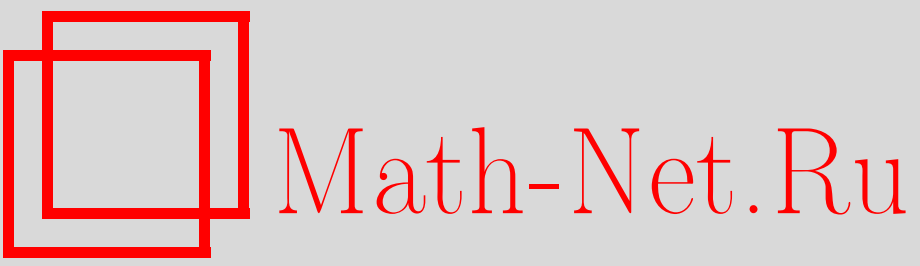

Е. Н. Огородников, Н. С. Яшагин, Постановка и решение задач типа Коши для дифференциальных уравнений второго порядка с дробными производными Римана-Лиувилля, Вестн. Сам. гос. техн. ун-та. Сер. Физ.мат. науки, 2010, выпуск 1(), 24-36

DOI: https://doi.org/10.14498/vsgtu771

Использование Общероссийского математического портала Math-Net.Ru подразумевает, что вы прочитали и согласны с пользовательским соглашением http: //www. mathnet.ru/rus/agreement

Параметры загрузки:

IP: 54.198 .187 .58

26 апреля 2023 г., 11:17:03 
УДК 517.968.72

\section{ПОСТАНОВКА И РЕШЕНИЕ ЗАДАЧ ТИПА КОШИ ДЛЯ ДИФФЕРЕНЦИАЛЬНЫХ УРАВНЕНИЙ ВТОРОГО ПОРЯДКА С ДРОБНЫМИ ПРОИЗВОДНЫМИ РИМАНА-ЛИУВИЛЛЯ}

\section{Е.Н. Огородников, Н.С. Яшагин}

Самарский государственный технический университет, 443100, Самара, ул. Молодогвардейская, 244.

E-mails: eugen.ogo@gmail.com; nik-yashagin@yandex.ru

Для двух линейных обыкновенных дифберенииалъных уравнений второго порядка с дробными производными Римана-Лиувилля обоснована корректность задач Коши соответственно в локальной (классической) и нелокальной постановках. Решения найдены в явном виде в терминах некоторых специальных функиий, связанных с функиией типа Миттаг-Лефблера. Отмечена непрерывная зависимость найденных решений от параметра дробности $\beta$. Для второго из рассмотренных уравнений предложена видоизмененная постановка задачи типа Коши, совпадаюшая с классической при $\beta=0$. Указанные дифференциальные уравнения предложены в качестве модельных уравнений дробных осиилляторов.

Ключевые слова: дробное исчисление, обыкновенные дифберенциалъные уравнения с дробными производными Римана-Лиувилля, дробные осиилляиионные уравнения, задача типа Коши, специальные функции типа Миттаг-Лебблера.

Введение. Уравнения, в которых неизвестная функция $y(x)$ присутствует под знаком производных дробного порядка, принято называть обыкновенными дифференциальными уравнениями дробного порядка [1]. Предполагается, что собственно порядок дифференциального уравнения, который, как обычно, определяется порядком старшей производной, может быть, в частности, и целым числом. Производные $D^{\alpha_{i}}$, где $\alpha_{i}$ в общем случае-комплексное число, в свою очередь, могут быть как производными Римана-Лиувилля, так и производными Маршо, Капуто, Эрдейи-Кобера и др.

В зарубежной математической литературе [2-4] сложился устойчивый термин «дробные дифференциальные уравнения» (fractional differential equations). Представляется, что термин «дифференциальные уравнения с дробными производными» более точно отражает суть предмета.

$\mathrm{K}$ настоящему моменту времени накоплено огромное количество материалов, относящихся к теории и приложениям дифференциальных уравнений с дробными производными. Прежде всего, отметим монографию А. A. Kilbas, H. M. Srivastava, J. J. Trujillo [2], в которой отражены основные теоретические результаты и методы решения, приведено большое количество примеров, а библиографический список содержит более девятисот источников, опубликованных к 2006 году. Теории и методам решения дифференциальных уравнений с дробными производными посвящены работы I. Podlubny [3], K. S. Miller, B. Ross [4]. Обстоятельное изложение основ дробного исчисления и некоторые его приложения к дифференциальным (обыкновенным и с частными производными) и интегральным уравнениям помимо [1] содержатся также в мо-

Евгений Николаевич Огородников (к.ф.-м.н., доцент), доцент, каф. прикладной математики и информатики. Николай Сергеевич Яшагин, аспирант, каф. прикладной математики и информатики. 
нографиях А. М. Нахушева [5,6] и Н. О. Вірченко, В. Я. Рибак [7]. Отметим также исторически первую монографию К. B. Oldham, J. Spanier [8], целиком посвященную дробному исчислению и некоторым его приложениям.

Неослабевающий поток публикаций по различным вопросам теории и приложений дифференциальных уравнений с дробными производными в периодической печати и материалах конференций позволяет сделать вывод о том, что это направление дробного исчисления продолжает интенсивно развиваться, а разработка его теории, по-видимому, еще далека от своего завершения. Не секрет, что столь повышенный интерес к дробному исчислению не случаен, а связан с многочисленными приложениями его в задачах реологии, вязкоупругости, аномальной диффузии в пористых (фрактальных) структурах, теории автоматического управления, физической химии, биологии и т. д.

В монографии [2] сформулированы и доказаны теоремы существования и единственности задач типа Коши для дифференциальных уравнений дробного (точнее произвольного) порядка самого общего вида:

$$
\left(D_{a+}^{\alpha} y\right)(x)=f\left[x, y(x),\left(D_{a+}^{\alpha_{1}} y\right)(x),\left(D_{a+}^{\alpha_{2}} y\right)(x), \ldots,\left(D_{a+}^{\alpha_{m}} y\right)(x)\right],
$$

где $\alpha, \alpha_{k} \in \mathbb{C}(k \equiv 1,2, \ldots, m), n-1<\operatorname{Re} \alpha<n(n=[\operatorname{Re} \alpha]+1$, при $\alpha \notin \mathbb{N}$ и $n=\alpha$, если $\alpha \in \mathbb{N}) ; 0<\alpha_{0}<\operatorname{Re} \alpha_{1}<\operatorname{Re} \alpha_{2}<\ldots<\operatorname{Re} \alpha_{m} ;\left(D_{a+}^{\alpha} y\right)(x)$, $\left(D_{a+}^{\alpha_{k}} y\right)(x)$ - левосторонние дробные производные Римана-Лиувилля порядка $\alpha$ и $\alpha_{k}$ соответственно.

Доказательства теорем опираются на идею редукции дифференциальных уравнений к соответствующим интегральным уравнениям типа Вольтерра второго рода и теорему Банаха о неподвижной точке. Указанная редукция осуществляется с помощью хорошо известного свойства дробных интегралов $I_{a+}^{\alpha}$ и производных $D_{a+}^{\alpha}$ Римана-Лиувилля, именно, если $f(x) \in L(a, b)$ и $f_{n-\alpha}(x)=\left(I_{a+}^{n-\alpha} f\right)(x) \in A C^{n}[a, b]$, то почти всюду на отрезке $[a, b]$ справедливо равенство

где

$$
\left(I_{a+}^{\alpha} D_{a+}^{\alpha} f\right)(x)=f(x)-\sum_{k=1}^{n} \frac{f_{n-\alpha}^{(n-k)}(a)}{\Gamma(\alpha-k+1)}(x-a)^{\alpha-k},
$$

$f_{n-\alpha}^{(n-k)}(a)=\lim _{x \rightarrow a+} \frac{d^{n-k}}{d x^{n-k}}\left[\left(I_{a+}^{n-\alpha} f\right)(x)\right]=\lim _{x \rightarrow a+}\left[\left(D_{a+}^{\alpha-k} f\right)(x)\right], \quad k \equiv 1,2, \ldots, n$.

Задание значений

$$
\lim _{x \rightarrow a+}\left[\left(D_{a+}^{\alpha-k} y\right)(x)\right]=b_{k}, \quad b_{k} \in \mathbb{R}, \quad k \equiv 1,2, \ldots, n
$$

для дифференциального уравнения и формирует так называемую задачу типа Коши.

ЗАмечание 1. Тождество для композиции $I_{a+}^{\alpha} D_{a+}^{\alpha}$, приведенное выше, в случае $0<\operatorname{Re} \alpha<1$ принимает вид

$$
\left(I_{a+}^{\alpha} D_{a+}^{\alpha} f\right)(x)=f(x)-\frac{f_{1-\alpha}(a)}{\Gamma(\alpha)}(x-a)^{\alpha-1},
$$

где $f_{1-\alpha}(x)=\left(I_{a+}^{1-\alpha} f\right)(x)$. 
Нетрудно показать, что в классе абсолютно непрерывных функций $A C[a, b]$ имеет место равенство

$$
\left(I_{a+}^{\alpha} D_{a+}^{\alpha} f\right)(x)=f(x) .
$$

Это равенство тем более справедливо для функций $f(x) \in C^{1}(a, b): f^{\prime}(x) \in$ $L(a, b)$.

В теоремах о существовании единственного решения заранее оговаривается класс функций, в котором это решение может быть найдено. В [2] рассмотрены различные функциональные пространства: $L^{\alpha}(a, b)$ - пространство функций с суммируемой производной $D_{a+}^{\alpha} y$, различные весовые пространства непрерывных функций $\mathrm{C}_{n-\alpha}^{\alpha}, \mathrm{C}_{n-\alpha, \gamma}^{\alpha}$, так или иначе обеспечивающие суммируемость старшей производной в дифференциальном уравнении.

Возникает вопрос: как формулировать задачу с начальными данными, не предполагая суммируемости старшей дробной производной?

Хорошо известно, что для дифференциальных уравнений с дробными производными Капуто ${ }^{C} D_{a+}^{\alpha}$ при определенных условиях корректна задача Коши в её классической постановке: $y^{(k)}(a)=b_{k}(k \equiv 1,2, \ldots, n)$. В некоторых работах высказывается мнение [9], что нелокальные начальные условия в задачах типа Коши для уравнений с дробными производными Римана-Лиувилля «не имеют чёткой физической интерпретации». В действительности математические модели динамических систем с памятью, диффузии в пористых средах и др. приводят именно к дифференциальным уравнениям с дробными производными Римана-Лиувилля. Отметим, что вместе с производными $D_{a+}^{\alpha} y$, $\alpha \in \mathbb{R}$, в дифференциальном уравнении могут быть и дробные производные $D_{a+}^{\alpha} y^{(n)}$ при $\alpha \in(0,1)$, однако таким уравнениям не уделяется достаточного внимания.

1. Пусть $T>0,[0, T]$ - конечный отрезок действительной числовой оси $\mathbb{R}$, $L(0, T) \stackrel{\text { def }}{=} L_{1}(0, T)$ - пространство суммируемых по Лебегу функций $f(t)$ (вообще говоря, комплекснозначных), $A C[0, T]$ - пространство абсолютно непрерывных на $[0, T]$ функций, а пространство $A C^{n}[0, T]$ состоит из функций $f(t)$ : $[0, T] \rightarrow \mathbb{C}, f(t) \in C^{n-1}[0, T]$, причём $f^{(n-1)}(t) \in A C[0, T]$, где $C^{n-1}[0, T]-$ множество функций непрерывно дифференцируемых до порядка $n-1$ включительно на отрезке $[0, T]$. Для левосторонних дробных производных Римана-Лиувилля $\left(I_{0+}^{\alpha} f\right)(t)$ и $\left(D_{0+}^{\alpha} f\right)(t)$ на отрезке $[0, T]$ всюду в работе используется не менее употребимое обозначение $I_{0 t}^{\alpha} f$ и $D_{0 t}^{\alpha} f$, причём если $\alpha>0$, то $D_{0 t}^{-\alpha} f \stackrel{\text { def }}{=} I_{0 t}^{\alpha} f$.

Уравнение

$$
\sum_{k=0}^{n} a_{k} D_{0 t}^{\alpha_{k}} \dot{u}+\sum_{k=0}^{m} b_{k} D_{0 t}^{\beta_{k}} u=f(t),
$$

где $u(t)$ - искомая, а $f(t)$ - заданная функции, $\dot{u}=d u / d t, t \in[0, T] ; a_{k}, b_{k} \in \mathbb{R}$, $\alpha_{k} \in(0,1], \beta_{k} \in[0,2)$, - является наиболее общей записью линейного неоднородного дифференциального уравнения порядка не выше двух с постоянными коэффициентами и левосторонними дробными производными РиманаЛиувилля от искомой функции и её первой производной $\dot{u}(t)$. Полагая в уравнении (1) $a_{0}=1$ при $\alpha_{0}=1$, приходим к дифференциальному уравнению 
второго порядка

$$
\ddot{u}+\sum_{k=1}^{n} a_{k} D_{0 t}^{\alpha_{k}} \dot{u}+\sum_{k=0}^{m} b_{k} D_{0 t}^{\beta_{k}} u=f(t)
$$

где $\alpha_{k} \in(0,1), \beta_{k} \in[0,2)$.

Уравнения (1) и (2) можно рассматривать в качестве модельных дифференциальных уравнений дробных осцилляторов [10]. Заметим сразу, что и постановка начальных задач, и структура их решения, и поведение в окрестности начальной точки $t=0$ существенно зависят от наличия или отсутствия в уравнениях (1) и (2) дробных производных искомой функции порядка $\beta_{k}>1$. Нетрудно показать, что при $\beta_{k} \in[0,1]$ и $f(t) \in L(0, T)$ для уравнения (2) корректна постановка классической задачи Коши с условиями

$$
u(0)=u_{0}, \quad \dot{u}(0)=\dot{u}_{0}
$$

Особенности постановки задач с начальными данными при $t=0$ и метод их решения ниже продемонстрируем на примере двух дифференциальных уравнений типа уравнения (2):

$$
\begin{gathered}
\ddot{u}+p D_{0 t}^{\beta} \dot{u}+q D_{0 t}^{2 \beta} u=f(t), \\
\ddot{u}+p D_{0 t}^{1+\beta} u+q D_{0 t}^{2 \beta} u=f(t),
\end{gathered}
$$

где $p, q \in \mathbb{R}, \beta \in(0,1 / 2), f(t) \in L(0, T)$ - заданная функция.

При $\beta=0$ уравнения (4) и (5) по определению дробной производной Римана-Лиувилля переходят в хорошо известное дифференциальное уравнение возмущенного осциллятора с вязким трением

$$
\ddot{u}+p \dot{u}+q u=f(t) \text {. }
$$

При $\beta \in(0,1)$ они являются математическими моделями, вообще говоря, существенно разных динамических процессов, т. к. в общем случае дробная производная $D_{0 t}^{1+\beta} u \neq D_{0 t}^{\beta} \dot{u}$. Действительно, даже для функций класса $A C^{2}[0, T]$ в силу достаточного признака существования дробной производной порядка $1<1+\beta<2$ и её определения [1] получим

$$
D_{0 t}^{1+\beta} u=\frac{d}{d t} D_{0 t}^{\beta} u=\frac{d}{d t}\left[\frac{u(0) t^{-\beta}}{\Gamma(1-\beta)}+I_{0 t}^{1-\beta} \dot{u}\right]=\frac{u_{0} t^{-(1+\beta)}}{\Gamma(-\beta)}+D_{0 t}^{\beta} \dot{u} .
$$

С другой стороны, на множестве функций $u(t): u(0)=u_{0}=0$ уравнения (4) и (5) совпадают, что позволяет использовать любое из них в качестве математической модели процесса возмущения осциллятора в состоянии покоя.

2. Рассмотрим вначале уравнение (4) с начальными условиями (3) и воспользуемся идеей редукции задачи к интегральному уравнению Вольтерра второго рода.

Прежде заметим, что решением рассматриваемых дифференциальных уравнений мы называем любую суммируемую функцию, обращающую уравнение в тождество почти всюду на отрезке $[0, T]$. А это значит, что решение 
уравнения (4) с условиями (3) следует искать в классе функций $A C^{2}[0, T]$, обеспечивающем существование почти всюду на $[0, T]$ суммируемой второй производной $\ddot{u}(t)$.

Имеет место следующее утверждение.

Лемма 1. Пусть $\beta \in(0,1 / 2), p, q \in \mathbb{R}, f(t) \in L(0, T), u(t) \in A C^{2}[0, T]$. Функиия и $(t)$ удовлетворяет почти всюду равенствам (3) и (4) тогда и только тогда, когда она удовлетворяет почти всюду интегральному уравнению

$$
u(t)+p I_{0 t}^{\alpha} u+q I_{0 t}^{2 \alpha} u=I_{0 t}^{2} f+u_{0}+\dot{u}_{0} t+p \frac{u_{0}}{\Gamma(1+\alpha)} t^{\alpha},
$$

где $\alpha=1-\beta$.

Доказ а тель ств в. Необходимость. Пусть $u(t) \in A C^{2}[0, T]$ удовлетворяет почти всюду равенствам (3) и (4). Проинтегрируем дважды левую и правую части равенства (4) при начальных условиях (3). Вычислим композиции операторов с учетом полугруппового свойства дробных интегралов [1] и замечания 1, сделанного во введении:

$$
\begin{gathered}
I_{0 t}^{2} D_{0 t}^{\beta} \dot{u}=I_{0 t}^{1} I_{0 t}^{1-\beta}\left(I_{0 t}^{\beta} D_{0 t}^{\beta} \dot{u}\right)=I_{0 t}^{1} I_{0 t}^{1-\beta} \dot{u}=I_{0 t}^{1-\beta} I_{0 t}^{1} \dot{u}=I_{0 t}^{1-\beta}\left(u-u_{0}\right)= \\
=I_{0 t}^{1-\beta} u-\frac{u_{0}}{\Gamma(1-\beta)} \int_{0}^{t} \frac{d \tau}{(t-\tau)^{\beta}}=I_{0 t}^{1-\beta} u-\frac{u_{0}}{\Gamma(1-\beta)} t^{1-\beta}, \\
I_{0 t}^{2} D_{0 t}^{2 \beta} u=I_{0 t}^{2-2 \beta}\left(I_{0 t}^{2 \beta} D_{0 t}^{2 \beta} u\right)=I_{0 t}^{2(1-\beta)} u,
\end{gathered}
$$

и, обозначая $1-\beta=\alpha$, получим равенство (7), что и доказывает необходимость.

Докажем достаточность. Пусть функция $u(t) \in A C^{2}[0, T]$ удовлетворяет интегральному уравнению (7). Продифференцируем равенство (7) по $t$ :

$$
\dot{u}(t)+p \frac{d}{d t} I_{0 t}^{\alpha} u+q \frac{d}{d t} I_{0 t}^{2 \alpha} u=I_{0 t}^{1} f+\dot{u}_{0}+p \frac{u_{0} t^{\alpha-1}}{\Gamma(\alpha)} .
$$

В силу достаточного признака существования дробной производной выполняется

поэтому

$$
\frac{d}{d t} I_{0 t}^{\alpha} u=D_{0 t}^{1-\alpha} u=\frac{u_{0}}{\Gamma(\alpha)} t^{\alpha-1}+I_{0 t}^{\alpha} \dot{u},
$$

$$
\dot{u}(t)+p I_{0 t}^{\alpha} \dot{u}+q I_{0 t}^{2 \alpha-1} u=I_{0 t}^{1} f+\dot{u}_{0} .
$$

Дифференцируя равенство (8) ещё раз, получим

$$
\ddot{u}(t)+p D_{0 t}^{1-\alpha} \dot{u}+q D_{0 t}^{2(1-\alpha)} u=f(t) .
$$

Переобозначая $\alpha=1-\beta$, приходим к уравнению (4).

Теперь покажем, что равенства (3) также выполняются. Действительно, переходя к пределу при $t \rightarrow 0+$ в левой и правой частях равенств $(7)$ и $(8)$, получим, что $\lim _{t \rightarrow 0+} u(t)=u_{0}=u(0)$ и $\lim _{t \rightarrow 0+} \dot{u}(t)=\dot{u}_{0}=\dot{u}(0)$. Таким образом, достаточность доказана. 
Итак, на основании леммы 1 задача Коши для дифференциального уравнения (4) с условиями (3) эквивалентна интегральному уравнению Вольтерра (7) с абелевыми ядрами, решение которого заведомо существует и единственно [11].

Нетрудно показать, что интегральный оператор $I+p I_{0 t}^{\alpha}+q I_{0 t}^{2 \alpha}$, где $I-$ тождественный оператор, в силу полугруппового свойства дробных интегралов допускает следующую факторизацию:

$$
I+p I_{0 t}^{\alpha}+q I_{0 t}^{2 \alpha}=\left(I-\lambda_{1} I_{0 t}^{\alpha}\right)\left(I-\lambda_{2} I_{0 t}^{\alpha}\right)
$$

где $\lambda_{1}, \lambda_{2}-$ корни многочлена $\lambda^{2}+p \lambda+q$. Тогда, обозначая правую часть интегрального уравнения (7) за $\varphi(t)$ и вводя новую функцию $v(t)=u(t)-$ $-\lambda_{2} I_{0 t}^{\alpha} u$, приводим уравнение (7) к равносильной ему системе интегральных уравнений

$$
\left(I-\lambda_{1} I_{0 t}^{\alpha}\right) v=\varphi(t), \quad\left(I-\lambda_{2} I_{0 t}^{\alpha}\right) u=v(t),
$$

решение которой начнём с первого уравнения.

Оба уравнения системы (9) являются хорошо известными уравнениями Вольтерра второго рода с ядром Абеля, и их решения выписываются в терминах функций типа Миттаг-Леффлера [12]. Используя далее интегральный оператор [13]

$$
\mathrm{E}_{a t ; \lambda}^{\alpha, \sigma} f=\int_{a}^{t}(t-\tau)^{\alpha-1} E_{\sigma}\left[\lambda(t-\tau)^{\sigma} ; \alpha\right] f(\tau) d \tau,
$$

где $\alpha, \sigma>0, \lambda \in \mathbb{C}$, запишем решение системы интегральных уравнений в виде

$$
v(t)=\varphi(t)+\lambda_{1} \mathrm{E}_{0 t ; \lambda_{1}}^{\alpha, \alpha} \varphi, \quad u(t)=v(t)+\lambda_{2} \mathrm{E}_{0 t ; \lambda_{2}}^{\alpha, \alpha} v .
$$

Тогда решением интегрального уравнения (7) является функция

$$
\begin{aligned}
u(t)=\varphi(t)+\lambda_{1} \mathrm{E}_{0 t ; \lambda_{1}}^{\alpha, \alpha} \varphi & +\lambda_{2} \mathrm{E}_{0 t ; \lambda_{2}}^{\alpha, \alpha}\left[\varphi(t)+\lambda_{1} \mathrm{E}_{0 t ; \lambda_{1}}^{\alpha, \alpha} \varphi\right]= \\
& =\varphi(t)+\lambda_{1} \mathrm{E}_{0 t ; \lambda_{1}}^{\alpha, \alpha} \varphi+\lambda_{2} \mathrm{E}_{0 t ; \lambda_{2}}^{\alpha, \alpha} \varphi+\lambda_{1} \lambda_{2} \mathrm{E}_{0 t ; \lambda_{2}}^{\alpha, \alpha} \mathrm{E}_{0 t ; \lambda_{1}}^{\alpha, \alpha} \varphi .
\end{aligned}
$$

Вычислим композицию операторов $\mathrm{E}_{0 t ; \lambda_{2}}^{\alpha, \alpha} \mathrm{E}_{0 t ; \lambda_{1}}^{\alpha, \alpha} \varphi$ с помощью тождества

$$
\mathrm{E}_{0 t ; \lambda_{2}}^{\mu, \alpha} \mathrm{E}_{0 t ; \lambda_{1}}^{\nu, \alpha} \varphi=\mathrm{E}_{0 t ; \lambda_{2}}^{\nu, \alpha} \mathrm{E}_{0 t ; \lambda_{1}}^{\mu, \alpha} \varphi=\mathrm{E}_{0 t ; \lambda_{1}, \lambda_{2}}^{\mu+\nu, \alpha, \alpha}
$$

где

$$
\mathrm{E}_{0 t ; \lambda_{1}, \lambda_{2}}^{\mu ; \alpha} \varphi=\int_{0}^{t}(t-\tau)^{\mu-1} \mathrm{E}_{\alpha, \beta}\left[\lambda_{1}(t-\tau)^{\alpha}, \lambda_{2}(t-\tau)^{\beta} ; \mu\right] \varphi(\tau) d \tau,
$$

введенный в работе [14] интегральный оператор с функцией типа МиттагЛеффлера двух аргументов:

$$
\mathrm{E}_{\alpha, \beta}(x, y ; \mu)=\sum_{k, n=0}^{\infty} \frac{x^{k} y^{n}}{\Gamma(k \alpha+n \beta+\mu)} .
$$


Используя свойства (11), (12), получим

$$
u(t)=\varphi(t)+\lambda_{1} \mathrm{E}_{0 t ; \lambda_{1}}^{\alpha, \alpha} \varphi+\lambda_{2} \mathrm{E}_{0 t ; \lambda_{2}}^{\alpha, \alpha} \varphi+\frac{\lambda_{1} \lambda_{2}}{\lambda_{1}-\lambda_{2}}\left(\lambda_{1} \mathrm{E}_{0 t ; \lambda_{1}}^{2 \alpha, \alpha} \varphi-\lambda_{2} \mathrm{E}_{0 t ; \lambda_{2}}^{2 \alpha, \alpha} \varphi\right) .
$$

Нам понадобится ещё одно легко проверяемое тождество для оператора $\mathrm{E}_{0 t ; \lambda}^{\alpha, \sigma}$. Именно, для любой суммируемой функции $\varphi(t)$ справедливо равенство

$$
\lambda \mathrm{E}_{0 t ; \lambda}^{2 \alpha, \alpha} \varphi=\mathrm{E}_{0 t ; \lambda}^{\alpha, \alpha} \varphi-I_{0 t}^{\alpha} \varphi .
$$

Действительно, решением интегрального уравнения $v(t)-\lambda I_{0 t}^{\alpha} v=\varphi(t)$ является функция $v(t)=\varphi(t)+\lambda \mathrm{E}_{0 t ; \lambda}^{\alpha, \alpha} \varphi$. Подставляя это решение в исходное уравнение и используя свойства композиции дробного интеграла с оператором $\mathrm{E}_{0 t ; \lambda}^{\alpha, \alpha}[15]$, получим

$$
\varphi(t)+\lambda \mathrm{E}_{0 t ; \lambda}^{\alpha, \alpha} \varphi-\lambda I_{0 t}^{\alpha} \varphi-\lambda^{2} I_{0 t}^{\alpha} \mathrm{E}_{0 t ; \lambda}^{\alpha, \alpha} \varphi=\varphi(t),
$$

откуда следует (13). Тогда

$$
\begin{aligned}
u(t)=\varphi(t)+\lambda_{1} \mathrm{E}_{0 t ; \lambda_{1}}^{\alpha, \alpha} \varphi+\lambda_{2} \mathrm{E}_{0 t ; \lambda_{2}}^{\alpha, \alpha} \varphi+\frac{\lambda_{1} \lambda_{2}}{\lambda_{1}-\lambda_{2}}\left(\mathrm{E}_{0 t ; \lambda_{1}}^{\alpha, \alpha} \varphi-\mathrm{E}_{0 t ; \lambda_{2}}^{\alpha, \alpha} \varphi\right)= \\
=\varphi(t)+\frac{\lambda_{1}^{2}}{\lambda_{1}-\lambda_{2}} \mathrm{E}_{0 t ; \lambda_{1}}^{\alpha, \alpha} \varphi-\frac{\lambda_{2}^{2}}{\lambda_{1}-\lambda_{2}} \mathrm{E}_{0 t ; \lambda_{2}}^{\alpha, \alpha} \varphi .
\end{aligned}
$$

Подставляя выражение для функции $\varphi(t)$ в равенство (14) с использованием некоторых свойств оператора (10) из работы [14] и известных свойств функции типа Миттаг-Леффлера [12], после несложных, но громоздких преобразований находим решение задачи (3), (4):

$$
\begin{aligned}
u(t)=u_{0}[- & \left.\frac{\lambda_{2}}{\lambda_{1}-\lambda_{2}} \mathrm{E}_{\alpha}\left(\lambda_{1} t^{\alpha} ; 1\right)+\frac{\lambda_{1}}{\lambda_{1}-\lambda_{2}} \mathrm{E}_{\alpha}\left(\lambda_{2} t^{\alpha} ; 1\right)\right]+ \\
& +\dot{u}_{0} t\left[\frac{\lambda_{1}}{\lambda_{1}-\lambda_{2}} \mathrm{E}_{\alpha}\left(\lambda_{1} t^{\alpha} ; 2\right)-\frac{\lambda_{2}}{\lambda_{1}-\lambda_{2}} \mathrm{E}_{\alpha}\left(\lambda_{2} t^{\alpha} ; 2\right)\right]+ \\
+\int_{0}^{t}(t-\tau)[ & {\left[\frac{\lambda_{1}}{\lambda_{1}-\lambda_{2}} \mathrm{E}_{\alpha}\left(\lambda_{1}(t-\tau)^{\alpha} ; 2\right)-\frac{\lambda_{2}}{\lambda_{1}-\lambda_{2}} \mathrm{E}_{\alpha}\left(\lambda_{2}(t-\tau)^{\alpha} ; 2\right)\right] f(\tau) d \tau . }
\end{aligned}
$$

В терминах дробной экспоненциальной функции [16]

$$
\operatorname{Exp}(\alpha, \mu ; \lambda ; t)=t^{\mu-1} \mathrm{E}_{\alpha}\left(\lambda t^{\alpha} ; \mu\right)
$$

это решение записывается так:

$$
\begin{aligned}
u(t)=u_{0} & {\left[-\frac{\lambda_{2}}{\lambda_{1}-\lambda_{2}} \operatorname{Exp}\left(\alpha, 1 ; \lambda_{1}, t\right)+\frac{\lambda_{1}}{\lambda_{1}-\lambda_{2}} \operatorname{Exp}\left(\alpha, 1 ; \lambda_{2}, t\right)\right]+} \\
& +\dot{u}_{0}\left[\frac{\lambda_{1}}{\lambda_{1}-\lambda_{2}} \operatorname{Exp}\left(\alpha, 2 ; \lambda_{1}, t\right)-\frac{\lambda_{2}}{\lambda_{1}-\lambda_{2}} \operatorname{Exp}\left(\alpha, 2 ; \lambda_{2}, t\right)\right]+
\end{aligned}
$$


$+\int_{0}^{t}\left[\frac{\lambda_{1}}{\lambda_{1}-\lambda_{2}} \operatorname{Exp}\left(\alpha, 2 ; \lambda_{1}, t-\tau\right)-\frac{\lambda_{2}}{\lambda_{1}-\lambda_{2}} \operatorname{Exp}\left(\alpha, 2 ; \lambda_{2}, t-\tau\right)\right] f(\tau) d \tau$.

Справедлива следующая теорема.

Теорема 1. Пусть в уравнении (4) $\beta \in(0,1 / 2) ; p, q \in \mathbb{R}, \lambda_{1}, \lambda_{2}$ - различные корни многочлена $\lambda^{2}+p \lambda+q ; f(t) \in L(0, T)$. Тогда единственное в классе функций $u(t) \in C^{1}[0, T] \cap C^{2}(0, T]$ решение задачи Коши (3) для уравнения (4) существует и может быть найдено по формуле (16).

Вместо формального доказательства ограничимся замечанием о том, что существование и единственность решения являются следствием эквивалентной редукции задачи Коши к интегральному уравнению типа Вольтерра второго рода и его однозначной разрешимости (см. лемму 1). С другой стороны, существование решения можно обосновать непосредственной подстановкой (16) в дифференциальное уравнение (4), что позволяет непосредственно убедиться в принадлежности найденного решения $u(t)$ классу функций $C^{1}[0, T] \cap C^{2}(0, T]$, причём $\ddot{u}(t)$ в точке $t=0$ имеет интегрируемую особенность порядка $2 \beta<1$.

Предельным переходом при $\lambda_{1} \rightarrow \lambda_{2}$ в формуле (16) нетрудно записать решение $u(t)$ для случая $\lambda_{1}, \lambda_{2} \in \mathbb{R}, \lambda_{1}=\lambda_{2}$. Случай, когда один из корней $\lambda^{2}+$ $+p \lambda+q$ равен нулю, получается непосредственно из (16). Если $\lambda_{1,2}=\sigma \pm \omega i$, то решение (16) принимает вид

$$
\begin{array}{r}
u(t)=u_{0}\left[\operatorname{Expc}(\alpha, 1 ; \lambda ; t)-\frac{\sigma}{\omega} \operatorname{Exps}(\alpha, 1 ; \lambda ; t)\right]+\frac{\dot{u}_{0}}{\omega} \operatorname{Exps}(\alpha ; 2-\alpha ; \lambda ; t)+ \\
+\frac{1}{\omega} \int_{0}^{t} \operatorname{Exps}(\alpha, 2-\alpha ; \lambda, t-\tau) f(t-\tau) d \tau,
\end{array}
$$

где $\lambda=\sigma+\omega i$, а введённые в работе [16] действительные функции

$$
\begin{aligned}
& \operatorname{Expc}(\alpha, \mu ; \lambda ; t)=t^{\mu-1} \operatorname{Ec}_{\alpha}\left(\lambda t^{\alpha} ; \mu\right)=\frac{1}{2} t^{\mu-1}\left[\mathrm{E}_{\alpha}\left(\lambda t^{\alpha} ; \mu\right)-\mathrm{E}_{\alpha}\left(\bar{\lambda} t^{\alpha} ; \mu\right)\right], \\
& \operatorname{Exps}(\alpha, \mu ; \lambda ; t)=t^{\mu-1} \operatorname{Es}_{\alpha}\left(\lambda t^{\alpha} ; \mu\right)=\frac{1}{2 i} t^{\mu-1}\left[\mathrm{E}_{\alpha}\left(\lambda t^{\alpha} ; \mu\right)-\mathrm{E}_{\alpha}\left(\bar{\lambda} t^{\alpha} ; \mu\right)\right]
\end{aligned}
$$

являются дробными аналогами функций $e^{\sigma t} \cos \omega t$ и $e^{\sigma t} \sin \omega t$, совпадая с последним при $\alpha=\mu=1$.

Функции (15) и (17) имеют простые формулы целочисленного дифференцирования [16] и связаны формулами, аналогичными формуле Эйлера

$$
\operatorname{Exp}(\alpha, \mu ; \lambda ; t)=\operatorname{Expc}(\alpha, \mu ; \lambda ; t)+i \operatorname{Exps}(\alpha, \mu ; \lambda ; t),
$$

в частности,

$$
\operatorname{Exp}(\alpha, 1 ; \lambda ; t)=\operatorname{Ec}_{\alpha}\left(\lambda t^{\alpha} ; 1\right)+i \operatorname{Es}_{\alpha}\left(\lambda t^{\alpha} ; 1\right) .
$$

Формулы дробного интегрирования и дифференцирования функции (15) будут следующими:

$I_{0 t}^{\alpha} \operatorname{Exp}(\sigma, \mu ; \lambda ; t)=\operatorname{Exp}(\sigma, \mu+\alpha ; \lambda ; t), \quad D_{0 t}^{\alpha} \operatorname{Exp}(\sigma, \mu ; \lambda ; t)=\operatorname{Exp}(\sigma, \mu-\alpha ; \lambda ; t)$, где $\alpha>0, \mu, \alpha$-произвольные комплексные параметры. 
Наконец, используем дробные аналоги функций $\cos z$ и $\sin z$ [16]:

$$
\begin{gathered}
\mathrm{C}_{\alpha}(z ; \mu)=\frac{1}{2}\left[\mathrm{E}_{\alpha}(i z ; \mu)-\mathrm{E}_{\alpha}(-i z ; \mu)\right]=\sum_{k=0}^{\infty} \frac{\left(-z^{2}\right)^{k}}{\Gamma(2 \alpha k+\mu)}, \\
\mathrm{S}_{\alpha}(z ; \mu)=\frac{1}{2 i}\left[\mathrm{E}_{\alpha}(i z ; \mu)-\mathrm{E}_{\alpha}(-i z ; \mu)\right]=z \sum_{k=0}^{\infty} \frac{\left(-z^{2}\right)^{k}}{\Gamma(2 \alpha k+\alpha+\mu)},
\end{gathered}
$$

для которых в случае $\lambda=i \omega$ справедлива формула Эйлера

$$
\operatorname{Exp}(\alpha, 1 ; i \omega, t)=\mathrm{C}_{\alpha}\left(\omega t^{\alpha} ; 1\right)+i \mathrm{~S}_{\alpha}\left(\omega t^{\alpha} ; 1\right) .
$$

Выпишем теперь решение задачи (3), (4) в случае чисто мнимых $\lambda_{1,2}=$ $= \pm \omega i$. Оно имеет вид

$$
\begin{aligned}
u(t)=u_{0} \mathrm{C}_{\alpha}\left(\omega t^{\alpha} ; 1\right)+\frac{\dot{u}_{0}}{\omega} t^{\beta} \mathrm{S}_{\alpha}\left(\omega t^{\alpha} ; 1+\beta\right)+ \\
\quad+\frac{1}{\omega} \int_{0}^{t}(t-\tau)^{\beta} \mathrm{S}_{\alpha}\left[\omega(t-\tau)^{\alpha} ; 1+\beta\right] f(\tau) d \tau,
\end{aligned}
$$

где $\beta=1-\alpha$. Хорошо видно, что при $\alpha=1$ функции $C_{1}(\omega t ; 1)=\cos \omega t$, $S_{1}(\omega t ; 1)=\sin \omega t$, и тогда $u(t)$ из $(18)$ является решением задачи Коши для дифференциального уравнения гармонического осциллятора (6) при $p=$ $=0$.

Рассмотрим, наконец, решение $u(t)$ в $(16)$ как функцию параметра $\alpha$. Справедливо утверждение.

Теорема 2. Решение задачи Коши (3) для дифференциалъного уравнения (4) является непреръьной функиией параметра $\alpha \in(1 / 2,1)$, причём при $\alpha \rightarrow 1(\beta \rightarrow 0)$ оно переходит в хорошо известное решение дифберенииального уравнения (6).

3. В этом разделе будет рассмотрено дифференциальное уравнение (5) в предположении, что его решение в начальной точке $t=0$ заведомо отлично от нуля, и тем самым одно из начальных условий: $u(0)=u_{0},-$ можно считать заданным.

Основная проблема постановки начальных задач для дифференциальных уравнений с дробными производными порядка больше единицы состоит в том, что даже для «достаточно хороших» функций дробная производная может оказаться функцией несуммируемой на отрезке $[0, T]$.

Запишем дифференциальное уравнение (5) в следующем виде:

$$
\frac{d}{d t}\left(\dot{u}+p D_{0 t}^{\beta} u+q I_{0 t}^{1-2 \beta} u\right)=f(t)
$$

Если ввести новую функцию $v(t)=\dot{u}+p D_{0 t}^{\beta} u+q I_{0 t}^{1-2 \beta} u$, то для простейшего дифференциального уравнения относительно функции $v(t)$ естественно предположить существование предела

$$
\lim _{t \rightarrow 0+} v(t)=\lim _{t \rightarrow 0+}\left(\dot{u}+p D_{0 t}^{\beta} u+q I_{0 t}^{1-2 \beta} u\right)=\lim _{t \rightarrow 0+}\left(\dot{u}+p D_{0 t}^{\beta} u\right)=u_{1} .
$$


Тогда $v(t)=u_{1}+I_{0 t}^{1} f$ при $f(t) \in L(0, T)$. Эти наводящие рассуждения приводят к следующей нелокальной постановке задачи типа Коши:

$$
u(0)=u_{0}, \lim _{t \rightarrow 0+}\left[\dot{u}(t)+p D_{0 t}^{\beta} u\right]=u_{1}
$$

для дифференциального уравнения (5) или (19).

Лемма 2. Пусть $\beta \in(0,1 / 2) ; p, q \in \mathbb{R}, f(t) \in L(0, T)$. Функиия $u(t) \in$ $C[0, T] \cap C^{2}(0, T]: \ddot{u}(t)+p D_{0 t}^{1+\beta} u \in L(0, T)$ удовлетворяет равенствам (5) и (20) тогда и только тогда, когда она удовлетворлет интегральному уравнению

$$
u(t)+p I_{0 t}^{\alpha} u+q I_{0 t}^{2 \alpha} u=I_{0 t}^{2} f+u_{0}+u_{1} t
$$

в каждой точке $(0, T]$, где $\alpha=1-\beta$.

До ка з а тель ст в о. Необходимость. Пусть $u(t) \in C[0, T] \cap C^{2}(0, T]$ удовлетворяет равенствам (5) и (20). Интегрируя уравнение (19) первый раз, получим

$$
\dot{u}(t)+p D_{0 t}^{\beta} u+q \frac{d}{d t} I_{0 t}^{1-2 \beta} u=I_{0 t}^{1} f+u_{1} .
$$

Второе интегрирование приводит к уравнению (21), так как для функций $u(t) \in C[0, T]$ выполняется $\lim _{t \rightarrow 0+} I_{0 t}^{1-2 \beta} u=\lim _{t \rightarrow 0+} I_{0 t}^{1-\beta} u=0$.

Достаточность очевидна в силу свойства $D_{0 t}^{\alpha} I_{0 t}^{\alpha} \varphi=\varphi(t)$, справедливого для любой функции $\varphi(t) \in L(0, T)$.

Осталось убедиться, что равенства (20) выполняются. Это тоже хорошо видно, если в уравнениях (21) и (22) перейти к пределу при $t \rightarrow 0+$.

Решение интегрального уравнения (21) проводиться по той же схеме, что и решение уравнения (7), и имеет вид (14), где следует подставить $\varphi(t)=$ $=I_{0 t}^{2} f+u_{0}+u_{1} t$. После необходимых вычислений получим решение задачи типа Коши (5)-(20) в виде

$$
\begin{aligned}
& u(t)=u_{0}\left[\frac{\lambda_{1}}{\lambda_{1}-\lambda_{2}} \mathrm{E}_{\alpha}\left(\lambda_{1} t^{\alpha} ; 1\right)-\frac{\lambda_{1}}{\lambda_{1}-\lambda_{2}} \mathrm{E}_{\alpha}\left(\lambda_{2} t^{\alpha} ; 1\right)\right]+ \\
& +u_{1} t\left[\frac{\lambda_{1}}{\lambda_{1}-\lambda_{2}} \mathrm{E}_{\alpha}\left(\lambda_{1} t^{\alpha} ; 2\right)-\frac{\lambda_{2}}{\lambda_{1}-\lambda_{2}} \mathrm{E}_{\alpha}\left(\lambda_{2} t^{\alpha} ; 2\right)\right]+ \\
& +\int_{0}^{t}(t-\tau)\left[\frac{\lambda_{1}}{\lambda_{1}-\lambda_{2}} \mathrm{E}_{\alpha}\left(\lambda_{1}(t-\tau)^{\alpha} ; 2\right)-\frac{\lambda_{2}}{\lambda_{1}-\lambda_{2}} \mathrm{E}_{\alpha}\left(\lambda_{2}(t-\tau)^{\alpha} ; 2\right)\right] f(\tau) d \tau,
\end{aligned}
$$

или

$$
\begin{gathered}
u(t)=u_{0}\left[\frac{\lambda_{1}}{\lambda_{1}-\lambda_{2}} \operatorname{Exp}\left(\alpha, 1 ; \lambda_{1}, t\right)-\frac{\lambda_{2}}{\lambda_{1}-\lambda_{2}} \operatorname{Exp}\left(\alpha, 1 ; \lambda_{2}, t\right)\right]+ \\
+u_{1}\left[\frac{\lambda_{1}}{\lambda_{1}-\lambda_{2}} \operatorname{Exp}\left(\alpha, 2 ; \lambda_{1}, t\right)-\frac{\lambda_{2}}{\lambda_{1}-\lambda_{2}} \operatorname{Exp}\left(\alpha, 2 ; \lambda_{2}, t\right)\right]+ \\
+\int_{0}^{t}\left[\frac{\lambda_{1}}{\lambda_{1}-\lambda_{2}} \operatorname{Exp}\left(\alpha, 2 ; \lambda_{1}, t-\tau\right)-\frac{\lambda_{2}}{\lambda_{1}-\lambda_{2}} \operatorname{Exp}\left(\alpha, 2 ; \lambda_{2}, t-\tau\right)\right] f(\tau) d \tau .
\end{gathered}
$$


Tеорема 3. Пусть в уравнении (5) $\beta \in(0,1 / 2) ; p, q \in \mathbb{R}, \lambda_{1}, \lambda_{2}-$ различные корни многочлена $\lambda^{2}+p \lambda+q ; f(t) \in L(0, T)$. Тогда единственное в классе функций $u(t) \in C[0, T] \cap C^{2}(0, T]$ решение задачи типа Коши (20) существует в форме равенства (23).

Как и в теореме 1 , доказательство существования решения легко проверить непосредственной подстановкой $u(t)$ из формулы (23) в дифференциальное уравнение (5). В процессе вычисления производных устанавливается принадлежность функции $u(t)$ классу $C[0, T] \cap C^{2}(0, T]$ и характер поведения её производных в окрестности точки $t=0$. Именно $\dot{u}(t)=O\left(t^{\alpha-1}\right), D_{0 t}^{\beta} u=$ $=O\left(t^{\alpha-1}\right), \alpha=1-\beta$, и сумма $\dot{u}(t)+D_{0 t}^{\beta} u$ есть функция, непрерывная в нуле, так как

$$
\lim _{t \rightarrow 0+}\left[\dot{u}(t)+p D_{0 t}^{\beta} u\right]=\left.\left[\dot{u}(t)+p D_{0 t}^{\beta} u\right]\right|_{t=0}=u_{1} .
$$

Вторая производная решения $\ddot{u}(t)$ имеет несуммируемую особенность в нуле, поскольку $\ddot{u}(t)=O\left(t^{\alpha-2}\right)$.

Справедливо утверждение, аналогичное теореме 2.

Теорема 4. Решение задачи Коши (20) для дифференциального уравнения (5) является непрерывной функиией параметра $\alpha \in(1 / 2,1)$, причём при $\alpha \rightarrow 1(\beta \rightarrow 0)$ оно переходит в известное решение дифференциального уравнения (6) с начальными условиями

$$
u(0)=u_{0}, \quad \lim _{t \rightarrow 0+}[\dot{u}(t)+p u(t)]=u_{1} .
$$

Вид второго начального условия в (24) подсказывает иную конструкцию второго условия в (20), обладающего тем свойством, что при $\beta \rightarrow 0$ оно переходит в классическое начальное условие $\lim _{t \rightarrow 0+} \dot{u}(t)=u_{2}$.

Рассмотрим видоизмененную задачу типа Коши для дифференциального уравнения (5)

$$
\lim _{t \rightarrow 0+} u(t)=u_{0}, \quad \lim _{t \rightarrow 0+}\left\{\dot{u}(t)+p\left[D_{0 t}^{\beta} u-u(t)\right]\right\}=u_{2} .
$$

Решение задачи с начальными условиями (25) очевидно существует, единственно и может быть найдено по формуле (23), в которой следует заменить $u_{1}$ на $p u_{0}+u_{2}$.

Действительно, если существует $\lim _{t \rightarrow 0+} u(t)=u_{0}$, то будет существовать и

$$
\lim _{t \rightarrow 0+}\left[\dot{u}(t)+p D_{0 t}^{\beta} u\right]=p u_{0}+u_{2}
$$

откуда $u_{1}=p u_{0}+u_{2}$.

При $\beta \rightarrow 0$ второе условие в (25) переходит в классическое, а решение уравнения (5) с условиями (25)

$$
u(t)=u_{0}\left[\frac{\lambda_{1}}{\lambda_{1}-\lambda_{2}} \operatorname{Exp}\left(\alpha, 1 ; \lambda_{1}, t\right)-\frac{\lambda_{2}}{\lambda_{1}-\lambda_{2}} \operatorname{Exp}\left(\alpha, 1 ; \lambda_{2}, t\right)\right]+
$$




$$
\begin{array}{r}
+\left(p u_{0}+u_{2}\right)\left[\frac{\lambda_{1}}{\lambda_{1}-\lambda_{2}} \operatorname{Exp}\left(\alpha, 2 ; \lambda_{1}, t\right)-\frac{\lambda_{2}}{\lambda_{1}-\lambda_{2}} \operatorname{Exp}\left(\alpha, 2 ; \lambda_{2}, t\right)\right]+ \\
+\int_{0}^{t}\left[\frac{\lambda_{1}}{\lambda_{1}-\lambda_{2}} \operatorname{Exp}\left(\alpha, 2 ; \lambda_{1}, t-\tau\right)-\frac{\lambda_{2}}{\lambda_{1}-\lambda_{2}} \operatorname{Exp}\left(\alpha, 2 ; \lambda_{2}, t-\tau\right)\right] f(\tau) d \tau
\end{array}
$$

при $\beta \rightarrow 0-$ в решение уравнения (6).

Заключение. Рассмотрены два обыкновенных дифференциальных уравнения второго порядка с дробными производными Римана-Лиувилля порядка меньше и больше единицы. Найдены точные решения задачи Коши и видоизмененной задачи типа Коши, обладающие тем свойством, что при устремлении параметра дробности $\beta$ к нулю и сами уравнения, и постановки начальных задач, и их решения переходят в классическую задачу и её решение для дифференциального уравнения осциллятора с вязким трением. Указанные дифференциальные уравнения могут выступать в качестве модельных уравнений дробных осцилляторов, описывающих некоторые динамические процессы, характерные для систем с памятью или сред с фрактальной структурой [5]. Однопараметрические семейства решений этих уравнений, в свою очередь, могут оказаться полезными в задачах аппроксимации и параметрической идентификации нелинейных динамических систем.

Работа выполнена при поддержке Федерального агентства по образованию (код проекта РНП 2.1.1/745).

\section{БИБЛИОГРАФИЧЕСКИЙ СПИСОК}

1. Самко С. Г., Килбас А. А., Маричев О.И. Интегралы и производные дробного порядка и некоторые их приложения. - Минск: Наука и техника, 1987. - 688 с.

2. Kilbas A.A., Srivastava H.M., Trujillo J.J. Theory and Applications of Fractional Differential Equations / North-Holland Mathematics Studies, 204; ed. J. van Mill. - Amsterdam: Elsevier, 2006. - 523 pp.

3. Podlubny I. Fractional Differential Equations: An Introduction to Fractional Derivatives, Fractional Differential Equations, Some Methods of Their Solution and Some of Their Applications / Mathematics in Science and Engineering, 198. — San Diego: Academic Press, 1999. - $340 \mathrm{pp}$.

4. Miller K.S., Ross B. An introduction to the fractional calculus and fractional differential equations. - New York: Jon Wiley \& Sons. Inc., 1993. - 366 pp.

5. Нахушев А. М. Дробное исчисление и его применение. - М.: Физматлит, 2003. - 272 с.

6. Нахушев A. М. Уравнения математической биологии. - М.: Высшая школа, 1995. $301 \mathrm{c.}$

7. Вірченко Н. О., Рибак В.Я. Основи дробового інтегро-дифференціровання. - Київ: Задруга, 2007. - 361 с.

8. Oldham K. B., Spanier J. The Fractional Calculus: Theory and Applications of Differentiation and Integration to Arbitrary Order / Mathematics in Science and Engineering, 111. San Diego: Academic Press, 1974. - 234 pp.

9. Чикрий А. А., Матичин И. И. Об аналоге формулы Коши для линейных систем произвольного дробного порядка // Доповіді Начіональної академіi наук України, 2007. № $1 .-$ C. $50-55$.

10. Огородников E. Н. Математические модели дробных осцилляторов, постановка и структура решения задачи Коши / В сб.: Тр. Шестой Всерос. научн. конф. с междунар. участием. Ч. 1: Дифференциальные уравнения и краевые задачи / Матем. моделирование и краевые задачи. - Самара: СамГТУ, 2009. - С. 177-181.

11. Tricomi F. G. Integral Eguations / Pure and Applied Mathematics, Vol. 5. - New York: Interscience, Inc., 1957. — 238 pp.; русск. пер.: Трикоми Ф. Интегральные уравнения. М.: Ин. лит., 1960. - 300 с.

12. Джрбашян M. М. Интегральные преобразования и представления функций в комплексной области. - М.: Наука, 1966. - 672 с. 
13. Огородников E.H. О некоторых краевых задачах для системы уравнений БицадзеЛыкова с инволютивной матрицей / В сб.: Тр. десятой межвуз. науч. конф. Ч. 3: Дифференциальные уравнения и краевые задачи / Мат. моделирование и краевые задачи. - Самара: СамГТУ, 2000. - С. 119-126.

14. Огородников E. Н., Яшагин Н. С. О некоторых свойствах операторов с функцией Миттаг-Леффлера в ядрах / В сб.: Тр. шестой Всероссийской научн. конф. с междунар. участием. Ч. 3: Дифференциальные уравнения и краевые задачи / Мат. моделирование и краевые задачи. - Самара: СамГТУ, 2009. - С. 181-188.

15. Огородников E.H. Корректность задачи Коши-Гурса для системы вырождающихся нагруженных гиперболических уравнений в некоторых специальных случаях и её равносильность задачам с нелокальными краевыми условиями // Вестн. Сам. гос. техн. ун-та. Сер. Физ.-мат. науки, 2004. - № 26. - С. 26-38.

16. Огородников E. H., Яшагин H. C. Некоторые специальные функции в решении задачи Коши для одного дробного осцилляционного уравнения // Вестн. Сам. гос. техн. унта. Сер. Физ.-мат. науки, 2009. - № 1(18). - С. 276-279.

Поступила в редакцию 01/II/2010; в окончательном варианте - 15/III/2010.

MSC: 45J05, 26A33

\section{SETTING AND SOLVING OF THE CAUCHY TYPE PROBLEMS FOR THE SECOND ORDER DIFFERENTIAL EQUATIONS WITH RIEMANN-LIOUVILLE FRACTIONAL DERIVATIVES}

\section{E. N. Ogorodnikov, N.S. Yashagin}

Samara State Technical University,

244, Molodogvardeyskaya str., Samara, 443100.

E-mails: eugen.ogo@gmail.com; nik-yashagin@yandex.ru

The correctness of the Cauchy problems in local (classical) and nonlocal staging for two linear ordinary second order differential equations with Riemann-Liouville fractional derivatives is substantiated. The explicit solutions in terms of some special functions related Mittag-Leffler type function are found out. Continuos dependence from the fractional parameter $\beta$ for these solutions is indicated. For the second equation the changing statement of the Cauchy type problem coinciding with classical when $\beta=$ $=0$ is studied. These equations are proposed such as some model fractional oscillating equation.

Key words: fractional calculus, ordinary differential equations with Riemann-Liouville fractional derivatives, fractional oscillating equation, Cauchy type problem, MittagLeffler type functions.

Original article submitted 01/II/2010; revision submitted $15 / \mathrm{III} / 2010$.

Eugeniy N. Ogorodnikov (Ph. D. (Phys. \& Math.)), Associate Professor, Dept. of Applied Mathematics \& Computer Science. Nikolay S. Yashagin, Postgraduate Student, Dept. of Applied Mathematics \& Computer Science. 